der zur Verbrennung gelangten Menge Substanz enthalten war, und zwar entspricht $1 \mathrm{ccm} \mathrm{1/2-Normalnatronlauge} \mathrm{0,001268} \mathrm{g}$ Phosphorsäure oder $0,0005536 \mathrm{~g}$ Phosphor.

Zur Orientierung bemerke ich, dass man am zweckmälsigsten von Pflanzensamen 0,5 bis $1 \mathrm{~g}$, von Fleisch, tierischen oder menschlichen Organen dieselbe Menge, von Blut zirka $5 \mathrm{ccm}$ verwendet. Ist die zur Fällung gelangte Menge Phosphorsäure zu gross, so geht die Filtration erheblich langsamer vor sich, und die Operation wird wesentlich zeitraubender, ohne an Genauigkeit zu gewinnen.

Flüssigkeiten dampft man zweckmälsig durch Kochen über kleiner Flamme in dem Verbrennungskolben auf ein Volumen von etwa $5 \mathrm{~cm}$ ein, ehe man die Säuremischung hinzugibt.

\title{
Hydrolyse des Exzelsins ${ }^{1}$, \\ Von
}

\section{Thomas B. Osborne und S. H. Clapp.}

Der grössere Teil der Proteïnsubstanz der Brasilnuss (Bertholletia exzelsa) besteht aus dem Globulin Exzelsin, das man in schönen, hexagonalen Kristallen erhalten kann, wenn man das Globulin langsam aus seiner Lösung abscheidet. Diese Kristalle haben keine Wirkung auf das polarisierte Licht und gehören nach Maschke offenbar zum regulären System. Da es möglich ist, eine grosse Menge dieses Globulins in vollständig homogener, kristallisierter Form zu erhalten, und da das Exzelsin auch innerhalb enger Grenzen vom Ammonsulfat gefällt wird, so hat man die Gelegenheit, ein Proteïnpräparat zu erhalten, das bessere Garantien für seine chemische Individualität bietet wie die amorphen Präparate anderer Proteine. Die Resultate der Hydrolyse des Exzelsins zeigen, wie die des kristallisierten Oxyhämoglobins aus Pferdeblut, dass das Exzelsin eben so viel Aminosäuren liefert, wie die meisten der anderen chemisch weniger gut definierten Proteïnpräparate. Das ölfreie Mehl der Brasilnuss wurde mit 3-prozentiger Ammonsulfatlösung ausgezogen, auf $50^{\circ}$ erhitzt und der vollständig klare Auszug dialysiert, bis sich der grössere Teil des gelösten Exzelsins in Kristallen niedergeschlagen hatte. Diese wurden nun gründlich mit verdünnter Koch-

1) Nach dem American Journal of Physiology Vol. XIX Nr. I, bearbeitet und übersetzt von Dr. V. Griessmayer. 
salzlösung und dann mit verdünntem Alkohol, der allmählich in der Stärke bis zum absoluten Alkohol gesteigert wurde, ausgewaschen. Das Präparat wurde dann über Schwefelsäure getrocknet. Von dem so dargestellten, kristallisierten Exzelsin wurden $500 \mathrm{~g}$, gleich $450 \mathrm{~g}$ wasserund aschefreier Substanz in einer Mischung von $500 \mathrm{ccm}$ Wasser und $500 \mathrm{ccm}$ Salzsäure vom sp. Gew. 1,19 suspendiert und in einem Wasserbad mit siedendem Wasser $2 \frac{1}{2}$ Stunden lang erwärmt. Das Erhitzen wurde dann in einem Ölbad fortgesetzt und die Lösung 18 Stunden lang gesotten. Die Esterifikation der Hydrochloride der Aminosäuren wurde dann in der oft beschriebenen Weise durchgeführt. Die dunkel gefärbten Ätherauszüge wurden in der gewohnten Weise mit Kaliumkarbonat und Natriumsulfat-Anhydrid getrocknet.

Die wässrige Schicht wurde von den anorganischen Salzen befreit und die Esterifikation wiederholt. Da die Ausbeute an Estern erheblich war, wurde der ganze Prozess wiederholt, aber diese letzte Behandlung lieferte sehr wenig ätherlöslichen Ester. Nach dem Abdestillieren des Äthers auf dem Wasserbade wurden die Ester unter vermindertem Drucke fraktioniert:

\begin{tabular}{cccc} 
Fraktion & Temp. des Bades & Druck & Gewicht \\
& bis zu & $m m$ & $g$ \\
I. & $51^{0}$ & $10,-$ & 43,55 \\
II. & $90^{0}$ & $10,-$ & 32,77 \\
III. & $105^{0}$ & 0,47 & 91,65 \\
IV. & $\mathbf{1 3 0 ^ { 0 }}$ & 0,40 & $\mathbf{5 9 , 5 7}$ \\
V. & $170^{0}$ & 0,38 & $\mathbf{5 6 , 8 3}$ \\
\cline { 3 - 4 } & & Total $\cdot .284,37$
\end{tabular}

Der undestillierte Rückstand wog $132 \mathrm{~g}$.

Fraktion I. Diese Fraktion bestand hauptsächlich aus Alkohol und Äther. Sie lieferte nach der Esterifikation mit Alkohol und Salzsäure $2,98 g$ Glylkokollester-Hydrochlorid, gleich $1,33 g$ Glykokoll. Der Schmelzpunkt war $144^{\circ}$.

Das Filtrat vom Glykokoll lieferte nach der Befreiung vom Chlor $1,75 \mathrm{~g}$ Alanin.

Fraktion II. Diese Fraktion wurde mit siedendem Wasser verseift, nnter vermindertem Drucke zur Trockne verdampft und das Prolin mit siedendem, absolutem Alkohol ausgezogen. Der in Alkohol unlösliche Teil, in gewöhnlicher Weise ersterifiziert; lieferte $2,59 \mathrm{~g}$ Glykokollester-Hydrochlorid vom Schmelzpunkte $144^{\circ}$, gleich $1,39 \mathrm{~g}$ Glykokoll. 
Das Filtrat vom Glykokoll warde vom Chlor befreit und einer systematischen, fraktionierten Kristallisation unterzogen. Man erhielt 3,23 $g$ Substanz von der Zusammensetzung der Aminovaleriansäure und $8,74 \mathrm{~g}$ Alanin.

Fraktion III. Diese Fraktion wurde mit 8 Volumen Wasser 6 Stunden lang gesotten, bis dië Lösung nicht mehr alkalisch gegen Lackmus reagierte. Die leicht gefärbte Lösung wurde dann unter vermindertem Drucke zur Trockne verdampft und das Prolin mit siedendem, absolutem Alkohol ausgezogen. Aus dem ungelöst bleibenden Teile erhielt man $39,15 \mathrm{~g}$ Leuzin. Die Substanz zersetzte sich bei $298^{\circ}$.

$0,1854 g$ Substanz gaben $0,3746 \mathfrak{g} \mathrm{CO}_{2}$ und $0,1647 g \mathrm{H}_{2} \mathrm{O}$.

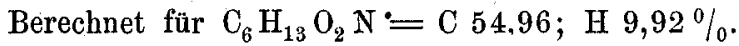

Gefunden . . . . C 55,$10 ; \mathrm{H} 9,87 \%$.

Die Substanz im Filtrat schien hauptsächlich aus Aminovaleriansäure zu bestehen, aber ihre Isolierung aus dieser Fraktion war so schwierig, dass man nur $0,98 g$ Substanz erhielt, die ganz gleichartig war.

$0,2155 \mathrm{~g}$ Substanz gaben $0,4032 g \mathrm{C} \mathrm{O}_{2}$ und $0,1862 g \mathrm{H}_{2} \mathrm{O}$.

Berechnet für $\mathrm{C}_{5} \mathrm{H}_{11} \mathrm{O}_{z} \mathrm{~N} \rightleftharpoons \mathrm{C} 51,28 ; \mathrm{H} \mathrm{9,4 \%}$.

Gefunden . . . . C 51,$03 ; \mathrm{H} 9,6^{\circ}{ }_{0}$.

Der Rückstand von dem Präparate an roher Aminovaleriansäure wog $2,57 \mathrm{~g}$. Beim Umkristallisieren aus verdünntem Alkohol gab es mit den berechneten Werten gut stimmende Zahlen.

Die alkohollöslichen Portionen der Fraktionen II und III bestanden hauptsächlich aus Prolin, wovon man $16,42 \mathrm{~g}$ erhielt. Um das aktive von dem razemischen zu unterscheiden, wurde das Kupfersalz angewendet und $3,7 \mathrm{~g}$ lufttrockenes, razemisches Prolinkupfer und $17,52 \mathrm{~g}$ des Links-Salzes, bei $110^{\circ}$ getrocknet, erhalten.

Das Phenylhydantoin des Linksprolins schmolz bei $143^{\circ}$.

Fraktion IV. Aus dieser Fraktion wurde der Phenylalaninester mit Äther ausgeschüttelt und durch Erwärmen mit starker Salzsäure verseift. Es wurden $6,74 \mathrm{~g}$ Phenylalanin als Hydrochlorid isoliert. $0,2331 \mathrm{~g}$ Substanz gaben $0,5576 \mathrm{~g} \mathrm{CO}_{2}$ und $0,1431 \mathrm{~g} \mathrm{H}_{2} \mathrm{O}$. Berechnet für $\mathrm{C}_{9} \mathrm{H}_{11} \mathrm{O}_{2} \mathrm{~N}=\mathrm{C} 65,45 ; \mathrm{H} 6,66 \%$. Gefunden . . . . . C 65,24; H 6,82\%.

Die wässrige Schicht lieferte nach dem Verseifen mit Baryt $7,77 \mathrm{~g}$ Assparaginsäure als Baryumsalz. 
$0,2159 g$ Substanz gaben $0,2868 g \mathrm{CO}_{2}$ und $0,1119 g \mathrm{H}_{2} \mathrm{O}$. $0,2947 \mathrm{~g}$ Substanz verlangten $3,15 \mathrm{ccm} 5 / 7 \mathrm{n}-\mathrm{H} \mathrm{Cl}$.

Berechnet für $\mathrm{C}_{4} \mathrm{H}_{7} \mathrm{O}_{4} \mathrm{~N}=\mathrm{C} 36,09 ; \mathrm{H} 5,26 ; \mathrm{N} 10,53 \%$. Gefunden . . . C C $36,22, \mathrm{H} 5,75 ; \mathrm{N} 10,68 \%$.

Das Filtrat vom Baryumaspartat, vom Baryt befreit, wurde mit Salzsäuregas gesättigt. Es schied sich bei $0^{0}$ keine Glutaminsäure als Hydrochlorid aus. Nach der Befreiung vom Chlor wurde der Rest der Asparaginsäure als Kupfersalz abgeschieden. Man erhielt 11,75g reinen Kupferaspartates, gleich $5,68 \mathrm{~g}$ Asparaginsäure.

$0,4321 \mathrm{~g}$ Substanz verlangten $2,28 \mathrm{~cm} 5 / 7 \mathrm{n}-\mathrm{H} \mathrm{Cl}$.

$0,4192 g$ Substanz gaben $0,1222 g \mathrm{Cu} \mathrm{O}$.

Berechnet für $\mathrm{C}_{4} \mathrm{H}_{5} \mathrm{O}_{4} \mathrm{NCu} .4^{1} / 2 \mathrm{H}_{2} \mathrm{O}=\mathrm{Cu} 23,07 ; \mathrm{N} 5,08 \%$. Gefunden . . . . . . . $\mathrm{Cu} 23,19 ; \mathrm{N} 5,27 \%$.

Fraktion V. Diese Fraktion wurde gerade so behandelt wie die vorige. Man erhielt 9,26 $g$ Phenylalanin als Hydrochlorid, 10,32 $g$ Glutaminsäure als Baryumsalz und $9,41 \mathrm{~g}$ als Hydrochlorid. Letzteres wurde in die freie Säure verwandelt, welche bei $202-203^{\circ}$ schmolz. Das Filtrat vom Glutaminsäurehydrochlorid lieferte weitere $8,03 \mathrm{~g}$ reines Kupferaspartat, gleich $3,88 \mathrm{~g}$ Asparaginsäure. Serin schien im Filtrate vom Kupferaspartat zugegen zu sein, wurde aber nicht in reinem Zustande isoliert.

Der Rückstand nach der Destillation.

Der Rückstand, der nach der Destillation der Ester übrig blieb, wog $132 \mathrm{~g}$. Er wurde in siedendem Alkohol gelöst und nach dem Abkühlen von der unlöslichen Substanz $(4,7 g)$ abfiltriert. Das Filtrat wurde zur Trockne verdampft und dadurch verseift, dass man es acht Stunden mit Baryt kochte. Man erhielt so $23,25 \mathrm{~g}$ GlutaminsäureHydrochlorid, gleich $18,62 g$ Glutaminsäure. Die freie Säure zersetzte sich bei $202-203^{\circ}$.

Man erhält so einen Gesamtgehalt an Glutaminsäure nach der Estermethode von $38,32 g$ oder $8,52 g$ des Exzelsins, was erheblich geringer ist wie die nach der direkten Metbode von $O$ sborne und Gilbert gefundenen $12,94 \%$.

Cystin.

Obwohl das Exzelsin ungefähr denselben Schwefelgehalt besitzt wie das Gliadin, so ist doch der Schwefelgehalt, den man als Sulfid erhält, 
wenn man das Exzelsin mit starker Natronlauge erhitzt, nur halb so gross wie der in ähnlicher Weise aus Gliadin erhaltene; auch konnte man kein Cystin bekommen, als man das rohe Tyrosin mit Merkurisulfat behandelte - wie beim Glatenin.

\section{Tyrosin.}

$100 \mathrm{~g}$ Exzelsin, gleich $90,19 \mathrm{~g}$ wasser- und aschefreiem Exzelsin, wurden mit $100 \mathrm{ccm}$ konz. Salzsäure und $100 \mathrm{ccm}$ Wasser 6 Stunden auf dem Ölbade gesotten. Nachdem man zur Sirupkonsistenz eingeengt und wiederholt mit Wasser unter stark vermindertem Druck eingedampft hatte, wurde der Rest der Salzsäure mit Natriumkarbonat neutralisiert. Nach dem Konzentrieren auf $400 \mathrm{~cm}$ wurde die Substanz, die sich ausschied, abfiltriert, das Filtrat weiter konzentriert und eine zweite Ausscheidung erhalten. Diese beiden Ausscheidungen wurden aus Wasser umkristallisiert, das erhaltene Produkt in 5-prozentiger Schwefelsäure gelöst und die Lösung mit Phosphorwolframsäure versetzt. Nach Entfernung des Phosphorwolframniederschlages wurden die Phosphorwolframsäure und die Schwefelsäure entfernt und zur Trockne eingedampft. Der Rückstand wurde mit siedender Eisessigsäure ausgezogen. Der Teil, der ungelöst zurückblieb, wog $2,83 \mathrm{~g}$, gleich $3,03 \%$. 0,2930 $\mathrm{g}$ Substanz, bei $100^{\circ}$ getrocknet, erforderten $2,24 \mathrm{ccm} 5 /{ }_{7} \mathrm{n}-\mathrm{H} \mathrm{Cl}$.

Berechnet für $\mathrm{C}_{9} \mathrm{H}_{13} \mathrm{O}_{3} \mathrm{~N}=\mathrm{N} 7,73 \%$.

Gefunden . . . . $\mathrm{N} 7,65 \%$.

\section{A rginin.}

$50 \mathrm{~g}$ Exzelsin, gleich $47,18 \mathrm{~g}$ wasser- und aschefreier Substanz, wurden hydrolysiert und das Arginin nach Kossel und Platten bestimmt. Die Lösung des Arginins enthielt Stickstoff, entsprechend 7,49 g Arginin. Setzt man noch $0,072 g$ für die Löslichkeit des Argininsilbers in der Lösung, aus der es ausfiel, zu, so haben wir 7,562 $g$ Arginin oder $16,02 \%$.

\section{Histidin.}

$500 \mathrm{~cm}$ der Lösung des Histidins, entsprechend $47,18 \mathrm{~g}$ trockenem und aschenfreiem Exzelsin, enthielten Stickstoff, entsprechend 0,6945 g Histidin oder $1,47 \%$.

$100 \mathrm{~cm}$ Lösung verlangten $3,77 \mathrm{~cm} 5 / 7 \mathrm{n}-\mathrm{H} \mathrm{Cl}=0,0377 \mathrm{~g} \mathrm{~N}$ in $100 \mathrm{ccm}$ oder $0,1885 \mathrm{~g}$ in $500 \mathrm{ccm}=0,6945 \mathrm{~g}$ Histidin. 
Dieses Histidin wurde in das Dichlorid, welches in den charakteristischen, rhomboëdrischen Kristallen kristallisierte, umgewandelt. Diese zersetzten sich bei $233^{\circ}$.

$0,0761 \mathrm{~g}$ Substanz gaben $0,0947 \mathrm{~g} \mathrm{Ag} \mathrm{Cl}$.

Berechnet für $\mathrm{C}_{6} \mathrm{H}_{11} \mathrm{O}_{2} \mathrm{~N}_{3} \mathrm{Cl}_{2}=\mathrm{N} 31,14 \%$. Gefunden . . . . . . N $30,76 \%$.

\section{Lysin.}

Das nach Kossel und Platten erhaltene Lysinpikrat aus $47,18 \mathrm{~g}$ Exzelsin wog 1,9884, gleich $0,7725 \mathrm{~g}$ Lysinpikrat oder $1,64 \%$.

$0,3 \mathrm{~g}$ Substanz, bei $110^{\circ}$ getrocknet, erforderten $5,6 \mathrm{ccm} 5 / 7 \mathrm{n}-\mathrm{H} \mathrm{Cl}$. Berechnet auf $\mathrm{C}_{6} \mathrm{H}_{34} \mathrm{O}_{2} \mathrm{~N}_{2} \cdot \mathrm{C}_{6} \mathrm{H}_{3} \mathrm{O}_{7} \mathrm{~N}_{3}=\mathrm{N} 18,70 \%$. Gefunden . . . . . . . N $18,66 \%$.

Resultate der Hydrolyse.

\begin{tabular}{|c|c|c|c|c|c|c|}
\hline Glykokoll & & $\begin{array}{l}\% \\
0,60\end{array}$ & Cystin & . & nicht & $\begin{array}{c}\% \\
\text { gefunden }\end{array}$ \\
\hline Alanin & . & 2,33 & Oxyprolin & & . $\ll$ & $\ll$ \\
\hline Aminovaleriansäure & $e$ & 1,51 & Tyrosin . & & . & 3,03 \\
\hline Leuzin . . . & . & 8,70 & Arginin . & . & . & 16,02 \\
\hline Prolin . . & . & 3,65 & Hystidin & & . & 1,47 \\
\hline Phenylalanin & . & 3,55 & Lysin . & & . & 1,64 \\
\hline Asparaginsäure & & 3,85 & Ammoniak & & & 1,80 \\
\hline Glutaminsäure & . . & . $\quad 12,94$ & Tryptophan & & . & anwesend \\
\hline Serin & nicht & gefunden & & tal & . & . 61,09 \\
\hline
\end{tabular}

Das Charakteristische dieser Exzelsinhydrolyse liegt darin, dass hièrbei ein aussergewöhnlich hoher Arginingehalt gefunden worden ist. 